\title{
Circulating miRNA Associated with Posttraumatic Stress Disorder in a Cohort of Military Combat Veterans
}

Christiana G. Martin ${ }^{a^{*}}$, Hyungsuk Kim ${ }^{\mathrm{a}}$, Sijung Yun ${ }^{\mathrm{b}}$, Whitney Livingston ${ }^{\mathrm{a}}$, Joseph Fetta ${ }^{\mathrm{a}}$, Vincent Mysliwiec ${ }^{\mathrm{c}}$, Tristin Baxter ${ }^{\mathrm{c}}$, Jessica M. Gill ${ }^{\mathrm{a}}$

${ }^{a}$ National Institutes of Nursing Research National Institutes of Health 10 Center Drive, Bethesda, MD 20892, USA

${ }^{\mathrm{b}}$ Yotta Biomed Potomac, MD 20854

${ }^{c}$ Madigan Army Medical Center 9040A Fitzsimmons Avenue, Tacoma, WA 98431, USA

*Co-corresponding author: Christiana G. Martin 9 Memorial Dr, Room 1S125 Bethesda, MD 20892 (301)451-2113 martinc2@nih.gov

*Co-corresponding author: Jessica M. Gill 1 Cloisters Court, Room 254 Bethesda, MD 20892 (301)451-8452 gillj@mail.nih.gov

\begin{abstract}
Posttraumatic stress disorder (PTSD) affects many returning combat veterans, but underlying biological mechanisms remain unclear. In order to compare circulating micro RNA (miRNA) of combat veterans with and without PTSD, peripheral blood from 24 subjects was collected following deployment, and isolated miRNA was sequenced. PTSD was associated with 8 differentially expressed miRNA. Pathway analysis shows that PTSD is related to the axon guidance and Wnt signaling pathways, which work together to support neuronal development through regulation of growth cones. PTSD is associated with miRNAs that regulate biological functions including neuronal activities, suggesting that they play a role in PTSD symptomatology.
\end{abstract}


miRNA Associated with PTSD in Military Combat Veterans 2

Keywords: PTSD; micro RNA; biomarker

\section{INTRODUCTION}

Posttraumatic stress disorder (PTSD) is a debilitating anxiety disorder with a disproportionate disease burden on military service members who deploy to combat, with rates between 10-30\% (Cohen et al., 2010; Tanielian \& Jaycox, 2008). PTSD is triggered by a lifethreatening event, such as combat, resulting in the onset of symptoms including intrusion, avoidance, negative alterations in cognition and mood, and alterations in arousal and reactivity ("Trauma- and Stressor-Related Disorders," 2013). Deployment itself can be a period of extreme stress which often includes unsafe living environments and physical injuries, placing individuals at high risk for PTSD. Additionally, military service members in recent conflicts have been at high risk for traumatic brain injuries (TBIs), as well as co-morbid symptoms of depression and sleep disorders along with PTSD (Kang, Yoon, \& Lyoo, 2015). Mild TBI (mTBI), a subtype of TBI classified by the Department of Defense as a non-penetrating TBI with a loss of 
miRNA Associated with PTSD in Military Combat Veterans 3

consciousness from 0-30 minutes, is by far the most common type of TBI experienced by service members, with mTBIs accounting for $82.4 \%$ of DoD reported TBIs in 2015 (2015 DoD TBI Worldwide Numbers 2016). The cumulative impact of deployment related disorders is estimated to be more than $\$ 3$ billion annually when considering only reduced productivity and missed work (Zhou et al., 2014). However, our ability to prevent the onset of PTSD and to treat it once symptoms present is limited, based in large part on our minimal understanding of the biological mechanisms related to the onset and maintenance of this disorder. Due to the complex biological nature of PTSD, investigating the molecular networks involved may provide valuable information on the pathogenesis of the disorder and therapeutic targets (Neylan, Schadt, \& Yehuda, 2014). Although promising biomarkers have been proposed, due to the multifaceted and heterogeneous nature of the disorder, a variety of biomarkers likely reflect separate aspects of mechanisms associated with PTSD (Jergovic et al., 2015; Michopoulos, Norrholm, \& Jovanovic, 2015). Thus, the exploration of novel biomarkers can expand our understanding of the biological processes effecting the disorder. Previous biomarker studies indicate the utility of using peripheral blood for approximating biological mechanisms within the central nervous system (Hayashi-Takagi, Vawter, \& Iwamoto, 2014; Kang et al., 2015), which is essential in clinical populations where obtaining cerebral spinal fluid is not plausible.

microRNAs (miRNAs) provides a new opportunity to understand biological underpinnings of PTSD. miRNAs, which are short, noncoding sequences that typically regulate gene expression by suppressing protein coding mRNA, have been demonstrated to be biomarkers for several psychological disorders, including schizophrenia, major depressive disorder, and bipolar disorder (Kichukova, Popov, Ivanov, \& Vachev, 2016; Maffioletti et al., 2016; Schmidt, Keck, \& Buell, 2015). Within the central nervous system, miRNA are considered to play a major 
role in gene expression regulation (Kuss \& Chen, 2008; Martinetz, 2016); however, the specific role of miRNAs in PTSD remains largely unknown. Previous studies link differential gene expression in the peripheral blood to PTSD in multiple clinical populations, including military and civilians (Glatt et al., 2013; Neylan et al., 2011; Segman et al., 2005; Tylee et al., 2015; Zieker et al., 2007), indicating that altered activity of genes relates to PTSD. Preclinical models of PTSD like behaviors have linked circulating miRNAs to neuronal activity, suggesting circulating miRNA in the periphery provide vital insights into central activity related to PTSD, and may serve as a potential biomarker (Balakathiresan et al., 2014). In an array-based clinical study, 18 dysregulated miRNA in veterans with PTSD have been reported, including miR-125a, which target genes in immune response pathways (Zhou et al., 2014). Two other downregulated miRNA, miR-3130-5p and miR-212, which have previously been implicated in other neurological disorders, were identified in subjects with PTSD and high rates of depression through the use of RNA sequencing (Wingo et al., 2015). By identifying miRNAs with differential expression in subjects with PTSD, necessary insights into gene networks that relate to PTSD symptomatology may be identified, which will ultimately improve our understanding of the biological mechanisms involved in the etiology of PTSD and the necessary insights into pharmacological targets.

To better understand the role of miRNAs in PTSD symptoms, we compared military personnel with and without PTSD using next generation sequencing of total miRNA from blood samples. This approach was selected for its unbiased ability to identify miRNA related to PTSD without being limited to predicted miRNA of interest. We tested the hypothesis that expression of miRNA in peripheral blood of military personnel with PTSD would significantly differ from combat matched controls without PTSD. 
miRNA Associated with PTSD in Military Combat Veterans 5

\section{METHODS}

\subsection{Participants}

In this study, a sample of military personnel (24 males, age 33.25 years \pm 7.55 ) from a larger study on sleep (findings previously reported (Mysliwiec et al., 2013)) were evaluated for PTSD. Active duty military personnel who had returned from deployment with Operation Enduring Freedom or Operation Iraqi Freedom within 18 months were eligible for participation. Demographic characteristics analyzed in this study included age, race, education, body mass index (BMI), deployment history, and medication use. Medications that participants were taking at the time of their evaluation were assessed including antidepressants, atypical antipsychotics, benzodiazepines, non-benzodiazepine receptor agonists (NBDRA), prazosin, and narcotics.

\subsection{Assessment for PTSD, mTBI, and Depression}

PTSD symptoms were assessed at two time points using the PTSD Checklist Military Version (PCL-M), which has 17 symptom items that are consistent with the DSM-IV-TR. A score of 50 or higher on the PCL-M is accepted to approximate a clinician assessed diagnosis of PTSD for purposes of informing diagnosis in high risk populations (Betthauser, Bahraini, Krengel, \& Brenner, 2012; Forbes, Creamer, \& Biddle, 2001). Based on the symptom inventory, subjects were classified into the PTSD (PCL-M>50) or control group (PCL-M<50). The Warrior Administered Retrospective Casualty Assessment Tool (WARCAT) was used to determine if a TBI occurred during the most recent deployment. To be included as a control, participants could not have any reported concussion or other head injury within the previous two years. Depression symptoms were assessed using the 16 item Quick Inventory of Depressive Symptomatology (QIDS) questionnaire (Trivedi et al., 2004). 
miRNA Associated with PTSD in Military Combat Veterans 6

\subsection{Blood collection and miRNA Sequencing}

Blood samples were collected into PAXgene blood RNA tubes and processed with PAXgene blood miRNA kit (Qiagen, Valencia, USA) for miRNA extraction. Quality and quantity of extracted RNA was assessed using the NanoDrop 2000 spectrophotometer (Thermo Fisher Scientific, Wilmington, DE) and the Agilent Bioanalyzer 2100 Small RNA assay (Agilent Technologies, Inc., Santa Clara, USA). All samples were enriched for miRNA using standard recommendations for preparation of small RNA libraries protocol. Barcoded libraries were prepared with an input of 1-5 ng of enriched small RNA using the Ion Total RNA-Seq Kit v2 (Life Technologies, Carlsbad, USA). Template-positive Ion PGM ${ }^{\mathrm{TM}}$ Ion Sphere ${ }^{\mathrm{TM}}$ Particles were prepared for sequencing using Ion PGM TM Template OT2 200 Template Kit v2 with pooled libraries optimized to $14 \mathrm{pM}$. Libraries were sequenced on the Ion Torrent $\mathrm{PGM}^{\mathrm{TM}}$ using the Ion 316 Chip v2 (Life Technologies, Carlsbad, USA).

\subsection{Data Analysis}

Sequencing reads were generated and initially processed by Ion Torrent Suite software version 4.4.2. Low quality reads and polyclonal sequences were filtered from sequencing data, then raw data was trimmed to 22 base pairs from $5^{\prime}$ end and profiles were generated by aligning sequences to hg19 with BWA; then, we quantified using miRDeep2 v2.0.0.7 and annotated based on miRBase v20. The miRNA-seq data was deposited to GEO under GSE87768. Differential expression analysis was conducted on DESeq2 based on the False Discovery Rate (FDR) by Benjamini and Hochberg multiple testing correction method (Benjamini \& Hochberg, 1995). Target prediction and analysis was conducted using the miRWalk2.0 database.

\section{RESULTS}




\subsection{Demographics Characteristics}

There were no significant differences between age, race, education, BMI, number of deployments, or medication use of PTSD and control subjects (Table 1). At baseline, PCL-M scores for control subjects ranged from 17-24 (M=20.44, SD=2.24), while scores for PTSD subjects ranged from 52-82 (M=64.87, $\mathrm{SD}=8.62)$. The only group differences between control and PTSD subjects were mTBI exposure and depression symptoms, with PTSD subjects having higher incidence of mTBI and higher depression symptoms.

Table 1. Demographics at baseline based on PTSD group.

\begin{tabular}{lcccc}
\hline & $\begin{array}{c}\text { PTSD } \\
n=15\end{array}$ & $\begin{array}{c}\text { No-PTSD } \\
\text { Control } \\
n=9\end{array}$ & $\mathbf{x}^{2} / \mathbf{t}$ & $\mathbf{P}$ \\
\hline Age: mean (SD) & $31.53(8.433)$ & $36.11(4.99)$ & 1.473 & 0.155 \\
Race: $n$ Caucasian (\%) & $7(46.7)$ & $5(55.6)$ & 0.046 & 0.582 \\
Education: mean (SD) & $13.27(1.53)$ & $13.78(2.59)$ & 0.611 & 0.547 \\
BMI: mean (SD) & $29.32(3.98)$ & $31.17(3.50)$ & 1.152 & 0.262 \\
Number of Deployments: mean (SD) & $2.13(1.13)$ & $2.56(1.42)$ & 0.806 & 0.429 \\
Medication Use: $n$ (\%) & $8(53.3)$ & $4(44.4)$ & 0.178 & 0.5 \\
PCL-M Score: mean (SD)* & $64.87(8.62)$ & $20.44(2.24)$ & -18.909 & $<.001$ \\
QIDS Score: mean (SD)* & $16.67(2.77)$ & $4.56(2.96)$ & -10.11 & $<.001$ \\
mTBI: $n$ (\%) & $8(53.3)$ & $0(0)$ & 7.2 & 0.009 \\
\hline
\end{tabular}

\section{2. miRNA Sequencing}

Of the 2578 human mature miRNAs quantified, 8 miRNA sequences were differentially expressed in PTSD participants compared to controls using a corrected threshold of FDR $\leq .05$ (Table 2). These included 4 significantly upregulated miRNA, with a fold change greater than 1.5, and 4 significantly downregulated miRNA, with a fold change less than -1.5 (Table 2). 
miRNA Associated with PTSD in Military Combat Veterans 8

Table 2. Differentially expressed miRNA between PTSD and control groups at baseline.

\begin{tabular}{lrr}
\hline miRNA & Fold Change & Adjusted P-value \\
\hline miR-19a-3p & 2.9422 & $8.71 \mathrm{E}-09$ \\
miR-486-3p & -4.4924 & $2.26 \mathrm{E}-07$ \\
miR-128-3p & -1.5267 & 0.001375 \\
miR-15b-3p & -1.5629 & 0.007071 \\
miR-125b-5p & -1.6985 & 0.011608 \\
miR-101-3p & 2.1202 & 0.012907 \\
miR-20b-5p & 1.9618 & 0.012907 \\
miR-20a-5p & 2.0624 & 0.017448 \\
\hline
\end{tabular}

\subsection{Pathway Analysis}

Target prediction of differentially expressed miRNA returned 15 KEGG pathways significantly targeted - with adjusted $\mathrm{p} \leq .05$ - by 4 or more of 8 miRNA. (Table 3 ). Of the genes predictively targeted by the miRNA, a significant proportion of genes were classified as transcription factors according to Fisher's exact test, with a mean of $739.88(\mathrm{SD}=140.88)$ transcription factors targeted by each miRNA. Transcription factors targeted by miRNA included Transcription factor 7 (T-cell specific, HMG-box), Transcription factor 7 like 1, and Transcription factor 7 like 2 .

Table 3. Top significantly targeted KEGG pathways.

\begin{tabular}{lrlr}
\hline & Number of & & Number of \\
Pathway & miRNA targeting & Pathway & miRNA targeting \\
\hline Axon guidance & 8 & Endocytosis & 5 \\
Wnt signaling pathway & 8 & Prostate cancer & 5 \\
Pathways in cancer & 8 & Apoptosis & 4 \\
Adherens junction & 6 & Glioma & 4 \\
Colorectal cancer & 6 & Long term potentiation & 4
\end{tabular}


Neurotrophin signaling pathway

Pancreatic cancer

Chronic myeloid leukemia
6 MAPK signaling pathway

6 Small cell lung cancer

5
4

4

\section{DISCUSSION}

Our major finding is that PTSD is associated with 4 upregulated and 4 downregulated miRNAs compared to controls in a military cohort. These miRNAs were identified through an unbiased sequencing approach, and are thought to target genes, particularly transcription factors, related to learning and memory processes that are likely related to PTSD symptoms. We also report that these miRNA relate to the function of the axonal guiding and Wnt signaling pathways which may relate to PTSD symptomatology, providing novel insights in the biological mechanisms of PTSD.

Our reporting of reductions in $\mathrm{miR}-15 \mathrm{~b}$ and $\mathrm{miR}-125 \mathrm{~b}$ validates a previous report in a sample of veterans with PTSD (Zhou et al., 2014). miR-15b is involved in cell division, metabolism, stress response, and angiogenesis (Finnerty et al., 2010), and in clinical samples, is linked to increased risk for cancer and Alzheimer's disease (Kumar et al., 2013), suggesting that there are broad disruptions in cellular function in PTSD that likely have implications for psychological and physical health. miR-125a (an isoform in the miR-125 family) also relates to cancer risk, in-part through altering immune responses, including activation and regulation of the NFkB pathway (Graff, Dickson, Clay, McCaffrey, \& Wilson, 2012). We and others have reported that PTSD is associated with greater inflammatory activity including increased proinflammatory cytokines, disruption of the NF-kB network, and dysregulation in DNA methylation and gene expression of immune related genes in clinical studies of PTSD (Gill, Saligan, Woods, \& Page, 2009; Guardado et al., 2016; Rusiecki et al., 2013; Smith et al., 2011; 
Tylee et al., 2015; Wang \& Young, 2016). Increases in pro-inflammatory cytokine activity has implications to brain function, as well as morbidity risks, suggesting that these miRNAs may contribute to epidemiological studies that link PTSD to immune related morbidity risks, including type II diabetes, hypertension, and chronic pain.

We also report that miR-19a and miR128 are associated with PTSD in this samples, which in conjunction with preclinical studies of related miRNA in these families, provide some insights into neuronal changes that likely relate to PTSD. In a preclinical model of PTSD-like behaviors, miR-19b (an isoform in the miR-19 family with a sequence closely related to miR19a) was upregulated in both serum and amygdala tissue (Balakathiresan et al., 2014). miR-19b contributes to the regulation of genes associated with fear memory (Balakathiresan et al., 2014), suggesting that alterations in this and related micro-RNAs may relate to vulnerability to the onset, or maintenance of PTSD-like symptoms. Similarly, expression of miR128b, a brain specific miRNA in the miR128 family, was altered in a pre-clinical construct that used a fear learning/extinction model, showing the critical role of this gene in re-experiencing like symptoms, and vulnerability for the onset of PTSD like-behavior (Lin et al., 2011). Together, with our findings, these studies indicate a key role of miRNA in PTSD symptomatology.

All of the 8 miRNAs associated with PTSD in this study significantly targeted genes in both the axon guidance pathway and Wnt signaling pathway, indicating that peripheral blood may be used to understand more central activities related to PTSD. A hallmark of PTSD is reductions in total white matter volume, as well as disruptions in specific neuronal areas including the hippocampus and amygdala (Lopresto, Schipper, \& Homberg, 2016). Therefore, disruptions in maintenance of axonal guidance and neurogenesis are likely related to PTSD pathology, and our findings may provide insights into how changes in neuronal integrity relate 
to PTSD. Specifically, the axon guidance pathway is involved with the attraction and repulsion of neuronal growth cones, plays a key role in neuron connections, and is essential for neuronal viability through repair mechanisms (Kanehisa, Sato, Kawashima, Furumichi, \& Tanabe, 2016). The axon guidance pathway interacts with multiple networks in addition to the Wnt signaling pathway to maintain neuronal integrity, including two pathways we report here to be related to PTSD, the neurotrophin signaling pathway, and the MAPK signaling pathway (Guthrie, 2007). Thus, regulation of this complex pathway is likely essential for neuron growth and neuroplasticity. Furthermore, changes to brain regions observed in patients with PTSD may be related to regulation of axon guidance, connecting this pathway to overall development and maintenance of symptoms.

We also report that the Wnt signaling pathway is associated with PTSD in our sample, and in previous clinical studies has been linked to altered behavior and cognitive disorders (Maguschak \& Ressler, 2012) including depression, mania, anxiety, and Alzheimer's disease (Maguschak \& Ressler, 2012). The Wnt pathway relates to neuronal differentiation and development, similar to the axonal guidance pathway, but also acts specifically on growth cones to attract towards or repel away from other neurons (Kanehisa et al., 2016; Yam \& Charron, 2013). Disruptions in WNT family proteins result in impaired memory formation and consolidation in the hippocampus and amygdala, and the onset of PTSD like behaviors (Caronia, Wilcoxon, Feldman, \& Grove, 2010; Fortress \& Frick, 2016; Maguschak \& Ressler, 2011; Xu et al., 2015). The WNT/ $\beta$-catenin pathway act at both pre-synaptic and post-synaptic cells controlling the size of the growth cone and the density of vesicles and stem cell proliferation and neurogenesis (Maguschak \& Ressler, 2012). Pre-clinical studies link fear conditioning to dysregulation of WNT expression in the amygdala and the onset of anxiety-like behaviors 
miRNA Associated with PTSD in Military Combat Veterans 12

(Maguschak \& Ressler, 2011; Mahan \& Ressler, 2012). It may then be plausible that altered activity of the WNT pathway in-part relates to the neuronal changes related to PTSD, including volume reductions in both the amygdala and hippocampus (Morey et al., 2012; Vieweg et al., 2006).

In summary, in this study we report that differentially expressed miRNA are associated with PTSD, using a peripheral blood sample. These miRNAs have centration activities related to axon guidance and Wnt signaling, pathways that are linked to neurological processes related to PTSD in preclinical studies. Although this study provides some novel insights into PTSD related biomarkers, it was limited by a relatively small sample size $(\mathrm{N}=24)$ of military service members. Despite the small sample, significant differences were found in the baseline analysis at the FDR $<.05$ cutoff, including 4 miRNAs with FDR $<.01$. Although PTSD subjects with and without previous head injuries were balanced in this sample, more conclusions could be drawn from repeating the study in a larger population of service members with a control cohort matched on all co-occurring symptoms. Therefore our findings suggest that additional, larger studies are needed to better explicate the role of gene regulation by miRNA in PTSD, and that this line of research will provide novel insights into the mechanisms of PTSD vulnerability as well as symptom maintenance in subjects such as those reported here. 
Conflicts of Interest and Source of Funding:

No author has any conflicts of interest to disclose. This study was funded, in part, by the Center for Neuroscience and Regenerative Medicine (Grant 60855).

Acknowledgement:

This work utilized the computational resources of the NIH HPC Biowulf cluster.

(http://hpc.nih.gov)

The opinions and assertions in this manuscript are those of the authors and do not necessarily represent those of the Department of the Army, Department of Defense, U.S. Government, or the Center for Neuroscience and Regenerative Medicine.

\section{REFERENCES}

2015 DoD TBI Worldwide Numbers. (2016). Retrieved from http://dvbic.dcoe.mil/files/tbi-numbers/DoDTBI-Worldwide-Totals_2015_Aug-12-2016_v1.0_508_2016-09-20.pdf

Balakathiresan, N. S., Chandran, R., Bhomia, M., Jia, M., Li, H., \& Maheshwari, R. K. (2014). Serum and amygdala microRNA signatures of posttraumatic stress: fear correlation and biomarker potential. J Psychiatr Res, 57, 65-73. doi:10.1016/j.jpsychires.2014.05.020

Benjamini, Y., \& Hochberg, Y. (1995). Controlling the False Discovery Rate: A Practical and Powerful Approach to Multiple Testing. Journal of the Royal Statistical Society. Series B (Methodological), 57(1), 289-300. doi:doi: 10.2307/2346101

Betthauser, L. M., Bahraini, N., Krengel, M. H., \& Brenner, L. A. (2012). Self-report measures to identify post traumatic stress disorder and/or mild traumatic brain injury and associated symptoms in military veterans of Operation Enduring Freedom (OEF)/Operation Iraqi Freedom (OIF). Neuropsychol Rev, 22(1), 35-53. doi:10.1007/s11065-012-9191-4

Caronia, G., Wilcoxon, J., Feldman, P., \& Grove, E. A. (2010). Bone morphogenetic protein signaling in the developing telencephalon controls formation of the hippocampal dentate gyrus and modifies fear-related behavior. J Neurosci, 30(18), 6291-6301. doi:10.1523/jneurosci.055010.2010

Cohen, B. E., Gima, K., Bertenthal, D., Kim, S., Marmar, C. R., \& Seal, K. H. (2010). Mental health diagnoses and utilization of VA non-mental health medical services among returning Iraq and Afghanistan veterans. J Gen Intern Med, 25(1), 18-24. doi:10.1007/s11606-009-1117-3

Finnerty, J. R., Wang, W. X., Hebert, S. S., Wilfred, B. R., Mao, G., \& Nelson, P. T. (2010). The miR-15/107 group of microRNA genes: evolutionary biology, cellular functions, and roles in human diseases. J Mol Biol, 402(3), 491-509. doi:10.1016/j.jmb.2010.07.051

Forbes, D., Creamer, M., \& Biddle, D. (2001). The validity of the PTSD checklist as a measure of symptomatic change in combat-related PTSD. Behav Res Ther, 39(8), 977-986.

Fortress, A. M., \& Frick, K. M. (2016). Hippocampal Wnt Signaling: Memory Regulation and Hormone Interactions. Neuroscientist, 22(3), 278-294. doi:10.1177/1073858415574728

Gill, J. M., Saligan, L., Woods, S., \& Page, G. (2009). PTSD is Associated With an Excess of Inflammatory Immune Activities. Perspectives in Psychiatric Care, 45(4), 262-277. doi:10.1111/j.17446163.2009.00229.x

Glatt, S. J., Tylee, D. S., Chandler, S. D., Pazol, J., Nievergelt, C. M., Woelk, C. H., .. Tsuang, M. T. (2013). Blood-based gene-expression predictors of PTSD risk and resilience among deployed marines: a pilot study. Am J Med Genet B Neuropsychiatr Genet, 162B(4), 313-326. doi:10.1002/ajmg.b.32167 
miRNA Associated with PTSD in Military Combat Veterans 14

Graff, J. W., Dickson, A. M., Clay, G., McCaffrey, A. P., \& Wilson, M. E. (2012). Identifying functional microRNAs in macrophages with polarized phenotypes. J Biol Chem, 287(26), 21816-21825. doi:10.1074/jbc.M111.327031

Guardado, P., Olivera, A., Rusch, H. L., Roy, M., Martin, C., Lejbman, N., . . . Gill, J. M. (2016). Altered gene expression of the innate immune, neuroendocrine, and nuclear factor-kappa B (NF-kappaB) systems is associated with posttraumatic stress disorder in military personnel. J Anxiety Disord, 38, 9-20. doi:10.1016/j.janxdis.2015.12.004

Guthrie, S. (2007). Neurotrophic Factors: Are They Axon Guidance Molecules? In D. Bagnard (Ed.), Axon Growth and Guidance (pp. 81-94). New York, NY: Springer New York.

Hayashi-Takagi, A., Vawter, M. P., \& Iwamoto, K. (2014). Peripheral biomarkers revisited: integrative profiling of peripheral samples for psychiatric research. Biol Psychiatry, 75(12), 920-928. doi:10.1016/j.biopsych.2013.09.035

Jergovic, M., Bendelja, K., Savic Mlakar, A., Vojvoda, V., Aberle, N., Jovanovic, T., ... Vidovic, A. (2015). Circulating levels of hormones, lipids, and immune mediators in post-traumatic stress disorder a 3-month follow-up study. Front Psychiatry, 6, 49. doi:10.3389/fpsyt.2015.00049

Kanehisa, M., Sato, Y., Kawashima, M., Furumichi, M., \& Tanabe, M. (2016). KEGG as a reference resource for gene and protein annotation. Nucleic Acids Research, 44(Database issue), D457D462. doi:10.1093/nar/gkv1070

Kang, H. J., Yoon, S., \& Lyoo, I. K. (2015). Peripheral Biomarker Candidates of Posttraumatic Stress Disorder. Exp Neurobiol, 24(3), 186-196. doi:10.5607/en.2015.24.3.186

Kichukova, T. M., Popov, N. T., Ivanov, H. Y., \& Vachev, T. I. (2016). Circulating microRNAs as a Novel Class of Potential Diagnostic Biomarkers in Neuropsychiatric Disorders. Folia Med (Plovdiv), 57(3), 159-172. doi:10.1515/folmed-2015-0035

Kumar, P., Dezso, Z., MacKenzie, C., Oestreicher, J., Agoulnik, S., Byrne, M., . . Oda, Y. (2013). Circulating miRNA biomarkers for Alzheimer's disease. PLoS One, 8(7), e69807. doi:10.1371/journal.pone.0069807

Kuss, A. W., \& Chen, W. (2008). MicroRNAs in brain function and disease. Curr Neurol Neurosci Rep, 8(3), 190-197.

Lin, Q., Wei, W., Coelho, C. M., Li, X., Baker-Andresen, D., Dudley, K., ... Bredy, T. W. (2011). The brainspecific microRNA miR-128b regulates the formation of fear-extinction memory. Nature Neuroscience, 14(9), 1115-1117. doi:10.1038/nn.2891

Lopresto, D., Schipper, P., \& Homberg, J. R. (2016). Neural circuits and mechanisms involved in fear generalization: Implications for the pathophysiology and treatment of posttraumatic stress disorder. Neurosci Biobehav Rev, 60, 31-42. doi:10.1016/j.neubiorev.2015.10.009

Maffioletti, E., Cattaneo, A., Rosso, G., Maina, G., Maj, C., Gennarelli, M., . . Bocchio-Chiavetto, L. (2016). Peripheral whole blood microRNA alterations in major depression and bipolar disorder. $J$ Affect Disord, 200, 250-258. doi:10.1016/j.jad.2016.04.021

Maguschak, K. A., \& Ressler, K. J. (2011). Wnt Signaling in Amygdala-Dependent Learning and Memory. The Journal of Neuroscience, 31(37), 13057-13067. doi:10.1523/jneurosci.3248-11.2011

Maguschak, K. A., \& Ressler, K. J. (2012). A Role for WNT/B-Catenin Signaling in the Neural Mechanisms of Behavior. Journal of neuroimmune pharmacology : the official journal of the Society on Neurolmmune Pharmacology, 7(4), 763-773. doi:10.1007/s11481-012-9350-7

Mahan, A. L., \& Ressler, K. J. (2012). Fear conditioning, synaptic plasticity and the amygdala: implications for posttraumatic stress disorder. Trends in Neurosciences, 35(1), 24-35. doi:http://dx.doi.org/10.1016/j.tins.2011.06.007

Martinetz, S. (2016). MicroRNA's impact on neurotransmitter and neuropeptide systems: small but mighty mediators of anxiety. Pflugers Arch, 468(6), 1061-1069. doi:10.1007/s00424-016-1814-9 
Michopoulos, V., Norrholm, S. D., \& Jovanovic, T. (2015). Diagnostic Biomarkers for Posttraumatic Stress Disorder: Promising Horizons from Translational Neuroscience Research. Biol Psychiatry, 78(5), 344-353. doi:10.1016/j.biopsych.2015.01.005

Morey, R. A., Gold, A. L., LaBar, K. S., Beall, S. K., Brown, V. M., Haswell, C. C., . . McCarthy, G. (2012). Amygdala volume changes in posttraumatic stress disorder in a large case-controlled veterans group. Arch Gen Psychiatry, 69(11), 1169-1178. doi:10.1001/archgenpsychiatry.2012.50

Mysliwiec, V., Gill, J., Lee, H., Baxter, T., Pierce, R., Barr, T. L., . . Roth, B. J. (2013). Sleep disorders in US military personnel: a high rate of comorbid insomnia and obstructive sleep apnea. Chest, 144(2), 549-557. doi:10.1378/chest.13-0088

Neylan, T. C., Schadt, E. E., \& Yehuda, R. (2014). Biomarkers for combat-related PTSD: focus on molecular networks from high-dimensional data. Eur J Psychotraumatol, 5. doi:10.3402/ejpt.v5.23938

Neylan, T. C., Sun, B., Rempel, H., Ross, J., Lenoci, M., O'Donovan, A., \& Pulliam, L. (2011). Suppressed monocyte gene expression profile in men versus women with PTSD. Brain Behav Immun, 25(3), 524-531. doi:10.1016/j.bbi.2010.12.001

Rusiecki, J. A., Byrne, C., Galdzicki, Z., Srikantan, V., Chen, L., Poulin, M., . . Baccarelli, A. (2013). PTSD and DNA Methylation in Select Immune Function Gene Promoter Regions: A Repeated Measures Case-Control Study of U.S. Military Service Members. Front Psychiatry, 4, 56. doi:10.3389/fpsyt.2013.00056

Schmidt, U., Keck, M. E., \& Buell, D. R. (2015). miRNAs and other non-coding RNAs in posttraumatic stress disorder: A systematic review of clinical and animal studies. J Psychiatr Res, 65, 1-8. doi:10.1016/j.jpsychires.2015.03.014

Segman, R. H., Shefi, N., Goltser-Dubner, T., Friedman, N., Kaminski, N., \& Shalev, A. Y. (2005). Peripheral blood mononuclear cell gene expression profiles identify emergent post-traumatic stress disorder among trauma survivors. Mol Psychiatry, 10(5), 500-513, 425. doi:10.1038/sj.mp.4001636

Smith, A. K., Conneely, K. N., Kilaru, V., Mercer, K. B., Weiss, T. E., Bradley, B., . . Ressler, K. J. (2011). Differential immune system DNA methylation and cytokine regulation in post-traumatic stress disorder. Am J Med Genet B Neuropsychiatr Genet, 156b(6), 700-708. doi:10.1002/ajmg.b.31212

Tanielian, T. L., \& Jaycox, L. (2008). Invisible wounds of war: Psychological and cognitive injuries, their consequences, and services to assist recovery (Vol. 1): Rand Corporation.

. Trauma- and Stressor-Related Disorders. (2013). In A. P. Association (Ed.), Diagnostic and Statistical Manual of Mental Disorders (5th ed.).

Trivedi, M. H., Rush, A. J., Ibrahim, H. M., Carmody, T. J., Biggs, M. M., Suppes, T., . . Kashner, T. M. (2004). The Inventory of Depressive Symptomatology, Clinician Rating (IDS-C) and Self-Report (IDS-SR), and the Quick Inventory of Depressive Symptomatology, Clinician Rating (QIDS-C) and Self-Report (QIDS-SR) in public sector patients with mood disorders: a psychometric evaluation. Psychol Med, 34(1), 73-82.

Tylee, D. S., Chandler, S. D., Nievergelt, C. M., Liu, X., Pazol, J., Woelk, C. H., .. Tsuang, M. T. (2015). Blood-based gene-expression biomarkers of post-traumatic stress disorder among deployed marines: A pilot study. Psychoneuroendocrinology, 51, 472-494. doi:10.1016/j.psyneuen.2014.09.024

Vieweg, W. V., Julius, D. A., Fernandez, A., Beatty-Brooks, M., Hettema, J. M., \& Pandurangi, A. K. (2006). Posttraumatic stress disorder: clinical features, pathophysiology, and treatment. Am J Med, 119(5), 383-390. doi:10.1016/j.amjmed.2005.09.027

Wang, Z., \& Young, M. R. (2016). PTSD, a Disorder with an Immunological Component. Front Immunol, 7, 219. doi:10.3389/fimmu.2016.00219 
Wingo, A. P., Almli, L. M., Stevens, J. J., Klengel, T., Uddin, M., Li, Y., . . Ressler, K. J. (2015). DICER1 and microRNA regulation in post-traumatic stress disorder with comorbid depression. Nat Commun, 6, 10106. doi:10.1038/ncomms10106

Xu, N., Zhou, W. J., Wang, Y., Huang, S. H., Li, X., \& Chen, Z. Y. (2015). Hippocampal Wnt3a is Necessary and Sufficient for Contextual Fear Memory Acquisition and Consolidation. Cereb Cortex, 25(11), 4062-4075. doi:10.1093/cercor/bhu121

Yam, P. T., \& Charron, F. (2013). Signaling mechanisms of non-conventional axon guidance cues: the Shh, BMP and Wnt morphogens. Current Opinion in Neurobiology, 23(6), 965-973. doi:http://dx.doi.org/10.1016/j.conb.2013.09.002

Zhou, J., Nagarkatti, P., Zhong, Y., Ginsberg, J. P., Singh, N. P., Zhang, J., \& Nagarkatti, M. (2014). Dysregulation in microRNA expression is associated with alterations in immune functions in combat veterans with post-traumatic stress disorder. PLoS One, 9(4), e94075. doi:10.1371/journal.pone.0094075

Zieker, J., Zieker, D., Jatzko, A., Dietzsch, J., Nieselt, K., Schmitt, A., . . Gebicke-Haerter, P. J. (2007). Differential gene expression in peripheral blood of patients suffering from post-traumatic stress disorder. Mol Psychiatry, 12(2), 116-118. doi:10.1038/sj.mp.4001905 